Vietnam Journal of Mechanics, VAST, Vol.39, No. 4 (2017), pp. 303-313

DOI:10.15625/0866-7136/9234

\title{
DYNAMIC ANALYSIS OF TWO-LINK FLEXIBLE MANIPULATOR CONSIDERING THE LINK LENGTH RATIO AND THE PAYLOAD
}

\author{
Duong Xuan Bien ${ }^{1, *}$, Chu Anh $\mathrm{My}^{1}$, Phan Bui Khoi ${ }^{2}$ \\ ${ }^{1}$ Le Quy Don Technical University, Hanoi, Vietnam \\ ${ }^{2}$ Hanoi University of Science and Technology, Vietnam \\ *E-mail: xuanbien82@yahoo.com \\ Received February 16, 2017
}

\begin{abstract}
Dynamic modeling and analysis of flexible manipulators play an essential role in optimizing mechanical design parameters and control law of real robot systems. In this paper, a nonlinear dynamic model of a manipulator is formulated based on the Finite Element Method. To analyze the dynamic behavior effectively, a numerical simulation scheme is proposed by taking full advantages of MATLAB and SIMULINK toolboxes. In this manner, the effect of varying payload and link length ratio of the manipulator to its elastic displacement is dynamically taken into account. The simulation results show that the payload and length link ratio have significant influences on the elastic displacements of the system. In particular, a proper spectrum of the link length ratio, in which the flexural displacement of the end point of the manipulator is smallest, is demonstrated. To this end, the proposed methodology could be used further to select optimal geometric parameters for the links of new robot designs.
\end{abstract}

Keywords: Flexible link, nonlinear dynamic equations, varied payload, varied length of links.

\section{INTRODUCTION}

The optimization of structure and energy is essential in robot arm design. This issue mostly relates to mechanical structure, required workspace, control accuracy, and loading capability of the robot. Robots designed with larger workspace, slimmer and lighter structure, as well as higher payload capability are always expected by designers. For such robot designs, the elastic displacement of links (flexible links) could not be neglected as usual. The elastic displacements are the main reason causing errors in positioning of the end-effector of the robot. Its performance relates closely to the rigidity of the structure, power consumption, reachable workspace, and manipulation speed. In particular, the loading capability and the geometric parameters of links should be analysed with respect to the elastic property of the system. Therefore, modeling and analysis of the flexible manipulator play an important role for the robot design and control.

(C) 2017 Vietnam Academy of Science and Technology 
Years ago, a number of researches focused on the flexible manipulator. Based on the dynamic model, Wang [1,2] proposed a technique to investigate the maximum load carrying capacity of a robot manipulator given a dynamic robot trajectory. Korayem [3] studied on the dynamic load carrying capacity of a two-link flexible manipulator using finite element method and Pontryagin's Minimum principle. Wang and Russell [4] presented numerical results for payload varying with the corresponding optimum shape design. Gee and Lee [5] discussed the modeling and nonlinear dynamic equations of a one-flexible link manipulator based on finite element method in Lagrange approach with varied payload. The effects of payload on the dynamic characteristic of a planar two-link flexible manipulator with the Assumed Modes Method (AMM) are studied and discussed in [6]. Reddy [7] presented the nonlinear modeling of a one-link flexible manipulator based on AMM and LS-DYNA. The results of both are compared. The numerical simulation shows that the LS-DYNA model gives the smooth hub-angle profile. Alam [8] proposed modeling of motion of single-link flexible which has been formulated as minimization problems with 8-dimensional searching space by using FSR-GA to estimate parameters of model so as to minimize the prediction error between system output, measured data and model output at each time step. Jafari [9] presented the nonlinear dynamic analysis of a single-link flexible manipulators by using FEM. Khali Ibrahim [10] described the simulation and experimental a single-link flexible manipulator with varying payloads. Narayana [11] presented the modeling and control PID of single-link flexible manipulator using linear techniques. Pole placement and LQR are proposed in controller design. Peza-Solís [12] used finite differences method and AMM to modeling a single-link flexible manipulator. Sliding modes control law is used to track trajectory with continuous time variables. Undergoing large deformation, Korayem [13] has built the general formula to finding the maximum allowable dynamic load of geometrically nonlinear flexible link manipulators. The dynamic model is based on small deflection theory. Ata [14] studied the effect of different sets of initial and boundary conditions on the joints torques. The AMM is used for computing elastic displacement. Here and now, there are remaining issues which need be studied. The nonlinear dynamic modeling with fully components in equations of motion has not been mentioned yet. The effects of varying payload and link length ratios at the same time are not considered yet, too.

This paper presents an investigation on the nonlinear dynamic modeling of a twolink flexible manipulator. The dynamic equations of the system are derived and presented in Lagrangian formulation. In particular, the hub inertia, mass of rotors, payload, structural damping, Coriolis and centrifugal forces are taken into the modeling to obtain a full dynamic model. To analyze the dynamic behavior effectively, a numerical simulation scheme is proposed by taking full advantages of MATLAB and SIMULINK toolboxes. In this manner, the effect of varying payload and link length ratio of the manipulator to its elastic displacement is dynamically taken into account. The simulation results show that the payload and length link ratio have a significant influence on the elastic displacements of the system. In particular, a proper spectrum of the link length ratio, in which the flexural displacement of the end point of the manipulator is smallest, is demonstrated. To this end, the proposed methodology could be used further to select optimal geometric parameters for the links of new robot designs. 


\section{DYNAMIC MODELLING}

\subsection{Finite Element model}

In Finite Element Method (FEM) approach, the flexible link is considered as an assemblage of a finite number of small elements. The elements are assumed interconnected at certain points, known as nodes. For each finite element, the scalar kinetic and potential energy functions are formulated as functions of the generalized coordinate. In this work, we concern a two-link flexible manipulator which motions on horizontal plane as depicted in Fig. 1.

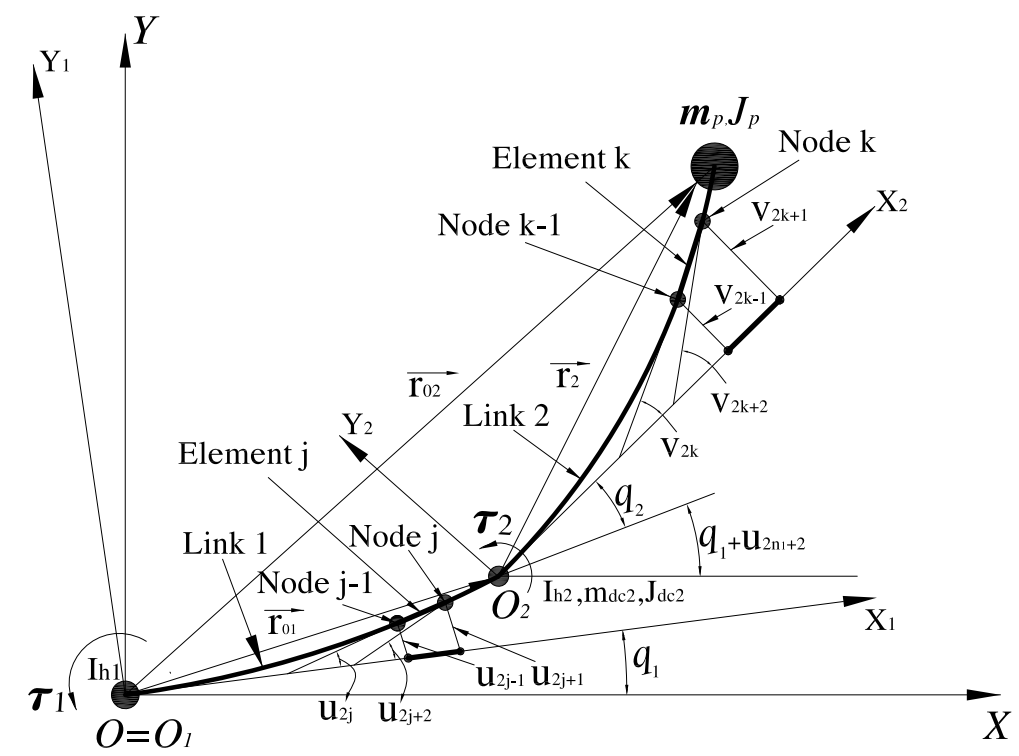

Fig. 1. Schematic diagram of a two-link flexible manipulator

The manipulator in Fig. 1 includes two flexible links. The rotation hubs at joint 1 and joint 2 are $q_{1}$ and $q_{2}$, respectively. The links are assumed as Euler-Bernoulli's beams. Each link can be divided into elements along the length of link and any element has 2 nodes. Each node has 2 variables which are the flexural and the slope displacement. Symbols $u_{2 j-1}, u_{2 j}, u_{2 j+1}$ and $u_{2 j+2}$ are flexural and slope at the first and the second nodes of element $j$. Similarly, $v_{2 k-1}, v_{2 k}, v_{2 k+1}$ and $v_{2 k+2}$ are flexural and slope at the first and the second nodes of $k$ element. The coordinate system XOY is the fixed frame. Coordinate system $X_{i} O_{i} Y_{i}$ is attached to link $i$. Symbols $\tau_{1}$ and $\tau_{2}$ are the applied torques at the joint 1 and 2. The vector $\mathbf{r}_{1}$ is from $O_{1}$ to the end point of link 1 in the $X_{1} O_{1} Y_{1}$. The vector $\mathbf{r}_{2}$ is from $\mathrm{O}_{2}$ to the end point of link 2 in the $\mathrm{X}_{2} \mathrm{O}_{2} Y_{2}$. The vectors $\mathbf{r}_{01}$ and $\mathbf{r}_{02}$ are from $O$ to end point of link 1 and link 2 in the XOY. $I_{h 2}, m_{d c 2}$ and $J_{d c 2}$ are the motor inertial moment, mass and inertial moment of rotor 2. Symbols $m_{P}$ and $J_{P}$ are the mass and inertial moment of payload on the end point of link 2. The material of links is assumed homogeneity. Link 1 is divided $n_{1}$ elements. The length of any element is $l_{j}$. Link 2 has $n_{2}$ elements and length of any element is $l_{k}$. Total elastic displacement $\mathbf{w}_{1 j}\left(x_{j}, t\right)$ of element 
$j$ with $\left(x_{j}, y_{j}\right)$ coordinate on $X_{1} O_{1} Y_{1}$ can be given by [5]

$$
w_{1 j}\left(x_{j}, t\right)=\mathbf{N}_{1 j}\left(x_{j}\right) \mathbf{Q}_{1 j}(t) .
$$

The generalized vector of shape function $\mathbf{N}_{1 j}\left(x_{j}\right)$ is defined as

$$
\mathbf{N}_{1 j}\left(x_{j}\right)=\left[\begin{array}{lllll}
x_{j} & \phi_{1}\left(x_{j}\right) & \phi_{2}\left(x_{j}\right) & \phi_{3}\left(x_{j}\right) & \phi_{4}\left(x_{j}\right)
\end{array}\right] .
$$

Mode shape function $\phi_{i}\left(x_{j}\right)$ can be presented in [3]. The generalized elastic displacement $\mathbf{Q}_{1 j}(t)$ is given as

$$
\mathbf{Q}_{1 j}(t)=\left[\begin{array}{lllll}
q_{1}(t) & u_{2 j-1} & u_{2 j} & u_{2 j+1} & u_{2 j+2}
\end{array}\right]^{T} .
$$

The coordinate $r_{1 j}$ of element $j$ on $X_{1} O_{1} Y_{1}$ is computed as

$$
\mathbf{r}_{1 j}=\left[\begin{array}{c}
(j-1) l_{j}+x_{j} \\
w_{1 j}\left(x_{j}, t\right)
\end{array}\right]
$$

where vector $\mathbf{r}_{01 j}=\mathbf{T}_{0}^{1} \mathbf{r}_{1 j}$ is coordinate of element $j$ on XOY and $\mathbf{T}_{0}^{1}=\left[\begin{array}{cc}\cos q_{1} & -\sin q_{1} \\ \sin q_{1} & \cos q_{1}\end{array}\right]$ is the transformation matrix from $X_{1} O_{1} Y_{1}$ to XOY system. Elastic kinetic energy of element $j$ is determined as

$$
T_{1 j}=\frac{1}{2} \int_{0}^{l_{j}} \rho_{1} A_{1}\left[\frac{\partial r_{01 j}}{\partial t}\right]^{2} d x_{j}=\frac{1}{2} \dot{\mathbf{Q}}_{1 j}^{T} \mathbf{M}_{1 j} \dot{\mathbf{Q}}_{1 j},
$$

where $\rho_{1}$ and $A_{1}$ are mass density and cross-sectional area of link 1 . Each element of inertial mass matrix $\mathbf{M}_{1 j}$ can be computed as

$$
\mathbf{M}_{1 j}(m, e)=\int_{0}^{l_{j}} \rho_{1} A_{1}\left[\frac{\partial \mathbf{r}_{01 j}}{\partial \mathbf{Q}_{1 j m}}\right]^{T}\left[\frac{\partial \mathbf{r}_{01 j}}{\partial \mathbf{Q}_{1 j e}}\right] d x_{j} ; \quad m, e=1,2, \ldots, 5 .
$$

The gravity effects can be ignored as the manipulator movement is confined to the horizontal plane. Defining $E_{1}$ and $I_{1}$ are Young's modulus and inertial moment of link 1 , the elastic potential energy of element $j$ is shown as $P_{1 j}$ with the stiffness matrix $\mathbf{K}_{1 j}$ and presented as [5]

$$
P_{1 j}=\frac{1}{2} \int_{0}^{l_{j}} E_{1} I_{1}\left[\frac{\partial^{2} w_{1 j}\left(x_{j}, t\right)}{\partial x_{j}^{2}}\right]^{2} d x_{j}=\frac{1}{2} \mathbf{Q}_{1 j}^{T}(t) \mathbf{K}_{1 j} \mathbf{Q}_{1 j}(t),
$$

where $u_{1}$ and $u_{2}$ elastic displacement at the first point, $u_{2 n+1}$ and $u_{2 n+2}$ are elastic displacement at the end point of link 1 , and vector $\mathbf{Q}_{1}(t)=\left[\begin{array}{llllll}q_{1} & u_{1} & u_{2} & \ldots & u_{2 n_{1}+1} & u_{2 n_{1}+2}\end{array}\right]^{T}$ represents the generalized coordinate of link 1 . The elastic kinetic energy and the potential energy of link 1 are yielded as

$$
T_{d h 1}=\sum_{j=1}^{n_{1}} T_{1 j}=\frac{1}{2} \dot{\mathbf{Q}}_{1}^{T}(t) \mathbf{M}_{d h 1} \dot{\mathbf{Q}}_{1}(t),
$$

and

$$
P_{1}=\sum_{j=1}^{n_{1}} P_{1 j}=\frac{1}{2} \mathbf{Q}_{1}^{T}(t) \mathbf{K}_{1} \mathbf{Q}_{1}(t)
$$


In above Eq. (8) and Eq. (9), the inertial mass matrix $\mathbf{M}_{d h 1}$ and the stiffness matrix $\mathbf{K}_{1}$ are constituted from matrices of elements follow FEM theory, respectively.

Similarly, the total elastic displacement $\mathbf{w}_{2 k}\left(x_{k}, t\right)$, the generalized shape function $\mathbf{N}_{2 k}\left(x_{k}\right)$ and the elastic displacement $\mathbf{Q}_{2 k}(t)$ of element $k$ with $\left(x_{k}, y_{k}\right)$ coordinate in $X_{2} \mathrm{O}_{2} Y_{2}$ can be written as

$$
\begin{aligned}
& w_{2 k}\left(x_{k}, t\right)=\mathbf{N}_{2 k}\left(x_{k}\right) \mathbf{Q}_{2 k}(t),
\end{aligned}
$$

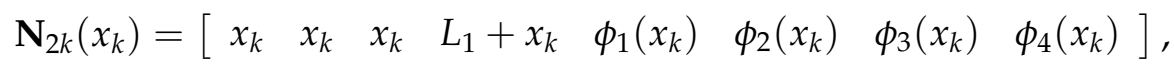

$$
\begin{aligned}
& \mathbf{Q}_{2 k}(t)=\left[\begin{array}{llllllll}
q_{1}(t) & u_{2 n_{1}+1} & u_{2 n_{1}+2} & q_{2}(t) & v_{2 k-1} & v_{2 k} & v_{2 k+1} & v_{2 k+2}
\end{array}\right]^{T} \text {. }
\end{aligned}
$$

Respectively, the coordinate $r_{2 k}$ of element $k$ on $\mathrm{X}_{2} \mathrm{O}_{2} Y_{2}$ and $\mathbf{r}_{02 k}$ on XOY are computed as

$$
\begin{gathered}
\mathbf{r}_{2 k}=\left[\begin{array}{c}
(k-1) l_{k}+x_{k} \\
w_{2 k}\left(x_{k}, t\right)
\end{array}\right], \\
\mathbf{r}_{02 k}=\mathbf{T}_{0}^{1}\left[\mathbf{r}_{1}+\mathbf{T}_{1}^{2} \mathbf{r}_{2 k}\right],
\end{gathered}
$$

where the transformation matrix $\mathbf{T}_{1}^{2}$ is given by [3]

$$
\mathbf{T}_{1}^{2}=\left[\begin{array}{cc}
\cos \left(q_{2}+u_{2 n_{1}+2}\right) & -\sin \left(q_{2}+u_{2 n_{1}+2}\right) \\
\sin \left(q_{2}+u_{2 n_{1}+2}\right) & \cos \left(q_{2}+u_{2 n_{1}+2}\right)
\end{array}\right]
$$

The elastic kinetic energy of element $k$ is determined as

$$
\mathbf{T}_{2 k}=\frac{1}{2} \int_{0}^{l_{k}} \rho_{2} A_{2}\left[\frac{\partial \mathbf{r}_{02 k}}{\partial t}\right]^{2} d x_{k}=\frac{1}{2} \dot{\mathbf{Q}}_{2 k}^{T} \mathbf{M}_{2 k} \dot{\mathbf{Q}}_{2 k} .
$$

Noted that each element of inertial mass matrix $M_{2 k}$ can be computed similarly (6). With the stiffness matrix $\mathbf{K}_{2 k}$ computed as in [15], the elastic potential energy of element $k$ is written as

$$
P_{2 k}=\frac{1}{2} \int_{0}^{l_{k}} E_{2} I_{2}\left[\frac{\partial^{2} w_{2 k}\left(x_{k}, t\right)}{\partial x_{k}^{2}}\right]^{2} d x_{k}=\frac{1}{2} \mathbf{Q}_{2 k}^{T}(t) \mathbf{K}_{2 k} \mathbf{Q}_{2 k}(t) .
$$

Similarly in link $1, v_{1}, v_{2}, v_{2 n_{2}+1}$ and $v_{2 n_{2}+2}$ are flexural and slope displacement at the first point and the end point of link 2, the generalized coordinate $\mathbf{Q}_{2}(t)$ of link 2 is defined as

$$
Q_{2}(t)=\left[\begin{array}{lllllllll}
q_{1} & u_{2 n_{1}+1} & u_{2 n_{1}+2} & q_{2} & v_{1} & v_{2} & \ldots & v_{2 n_{2}+1} & v_{2 n_{2}+2}
\end{array}\right]^{T} .
$$

The elastic kinetic energy and potential energy of link 2 are given by

$$
\begin{gathered}
T_{d h 2}=\sum_{k=1}^{n_{2}} T_{2 k}=\frac{1}{2} \dot{\mathbf{Q}}_{2}^{T}(t) \mathbf{M}_{d h 2} \dot{\mathbf{Q}}_{2}(t), \\
P_{2}=\sum_{k=1}^{n_{2}} P_{2 k}=\frac{1}{2} \mathbf{Q}_{2}^{T}(t) \mathbf{K}_{2} \mathbf{Q}_{2}(t) .
\end{gathered}
$$

The inertial mass matrix $\mathbf{M}_{d h 2}$ and the stiffness matrix $\mathbf{K}_{2}$ are calculated according to FEM theory. 


\subsection{Dynamic equations}

As noted above, the methodology is based upon FEM and Lagrange-Euler method. The main advantage of using a Lagrange formulation is that it is simple and systematic, which is of great importance when a computer is used. Fundamentally, the method relies on the Lagrange equations with Lagrange function $L=T-P$ are given by

$$
\frac{d}{d t}\left(\frac{\partial L}{\partial \dot{\mathbf{Q}}}\right)-\frac{\partial L}{\partial \mathbf{Q}}=\mathbf{F}(t)
$$

Vector $\mathbf{Q}$ represents the generalized coordinate overall system and is given as

$$
\mathbf{Q}=\left[\begin{array}{llllllllllll}
q_{1} & u_{1} & u_{2} & \ldots & u_{2 n_{1}+1} & u_{2 n_{1}+2} & q_{2} & v_{1} & v_{2} & \ldots & v_{2 n_{2}+1} & v_{2 n_{2}+2}
\end{array}\right]^{T} .
$$

$F(t)$ is the external generalized forces acting on specific generalized coordinate $\mathbf{Q}$. The overall system kinetic energy $T$ is computed as

$$
T=T_{d h 1}+T_{d c 1}+T_{m_{d}}+T_{d h 2}+T_{d c 2}+T_{m_{P}}=\frac{1}{2} \dot{Q}^{T}(t) M \dot{Q}(t),
$$

where $T_{d c 1}$ and $T_{d c 2}$ are kinetic energy of motor 1 and motor 2 . They are given as

$$
\begin{gathered}
T_{d c 1}=\frac{1}{2} I_{h 1} \dot{q}_{1}^{2}(t)=\frac{1}{2} \dot{\mathbf{Q}}_{1}^{T}(t) \mathbf{M}_{d c 1} \dot{\mathbf{Q}}_{1}(t), \\
T_{d c 2}=\frac{1}{2} I_{h 2}\left(\dot{q}_{1}+\dot{q}_{2}+\dot{u}_{2 n_{1}+2}\right)^{2}(t)=\frac{1}{2} \dot{\mathbf{Q}}_{2}^{T}(t) \mathbf{M}_{d c 2} \dot{\mathbf{Q}}_{2}(t) .
\end{gathered}
$$

The kinetic energy of mass of motor 2 at the end of link 1 and kinetic energy of payload at the end of link 2 are $T_{m d}$ and $T_{m P}$, respectively. They are computed as

$$
\begin{gathered}
T_{m d}=\frac{1}{2} m_{d c 2}\left[\frac{\partial \mathbf{r}_{01}}{\partial t}\left(L_{1}, t\right)\right]^{2}+\frac{1}{2} J_{d c 2}\left(\dot{q}_{1}+\dot{u}_{2 n_{1}+2}\right)^{2}=\frac{1}{2} \dot{\mathbf{Q}}_{1}^{T}(t) \mathbf{M}_{m d} \dot{\mathbf{Q}}_{1}(t), \\
T_{m P}=\frac{1}{2} m_{p}\left[\frac{\partial \mathbf{r}_{02}}{\partial t}\left(L_{2}, t\right)\right]^{2}+\frac{1}{2} J_{p}\left(\dot{q}_{1}+\dot{u}_{2 n_{1}+2}+\dot{q}_{2}+\dot{v}_{2 n_{2}+2}\right)^{2}=\frac{1}{2} \dot{\mathbf{Q}}_{2}^{T}(t) \mathbf{M}_{m P} \dot{\mathbf{Q}}_{2}(t) .
\end{gathered}
$$

The matrices $\mathbf{M}_{d c 1}, \mathbf{M}_{d c 2}, \mathbf{M}_{m d}$ and $\mathbf{M}_{m P}$ are easy determined following vectors of variables $\mathbf{Q}_{1}(t)$ and $\mathbf{Q}_{2}(t)$. The mass matrix $\mathbf{M}$ is sum of $\mathbf{M}_{1}$ and $\mathbf{M}_{2}$ which are mass matrix of link 1 and link 2, respectively. Each element in $\mathbf{M}$ is determined from FEM theory. Elements $(i, j)$ of $\mathbf{M}$ are computed following the algorithm in [16]. They are given as

$$
\begin{gathered}
\mathbf{M}_{1}=\mathbf{M}_{d h 1}+\mathbf{M}_{d c 1}+\mathbf{M}_{m d} \\
\mathbf{M}_{2}=\mathbf{M}_{d h 2}+\mathbf{M}_{d c 2}+\mathbf{M}_{m P} \\
\mathbf{M}[i, j]=\mathbf{M}_{1}\left[i_{1}, j_{1}\right]+\mathbf{M}_{2}\left[i_{2}, j_{2}\right] ;\left(i_{1}, j_{1}=1,2, \ldots, 2 n_{1}+3 ; i_{2}, j_{2}=1,2, \ldots, 2 n_{2}+6\right) .
\end{gathered}
$$

The matrix $\mathbf{K}$ is determined from $\mathbf{K}_{1}$ and $\mathbf{K}_{2}$ similarly $\mathbf{M}$. The overall system potential energy is computed as

$$
P=P_{1}+P_{2}=\frac{1}{2} \mathbf{Q}^{T}(t) \mathbf{K} \mathbf{Q}(t) .
$$

When kinetic and potential energies are known, it is possible to express (21) as shown

$$
\mathbf{M}(\mathbf{Q}) \ddot{\mathbf{Q}}+\mathbf{C}(\mathbf{Q}, \dot{\mathbf{Q}}) \dot{\mathbf{Q}}+\mathbf{D} \dot{\mathbf{Q}}+\mathbf{K Q}=\mathbf{F}(t),
$$


where structural damping $\mathbf{D}$ and Coriolis force $\mathbf{C}$ are calculated as

$$
\begin{gathered}
\mathbf{C}(\mathbf{Q}, \dot{\mathbf{Q}}) \dot{\mathbf{Q}}=\dot{\mathbf{M}}(\mathbf{Q}) \dot{\mathbf{Q}}-\frac{1}{2} \frac{\partial}{\partial \mathbf{Q}}\left(\dot{\mathbf{Q}}^{T} \mathbf{M}(\mathbf{Q}) \dot{\mathbf{Q}}\right), \\
\mathbf{D}=\alpha \mathbf{M}+\beta \mathbf{K},
\end{gathered}
$$

where $\alpha$ and $\beta$ are the damping ratios of the system which are determined by experiment. The nonlinear dynamic equations (32) are determined from (30), (33) and (34). The dynamic behaviors of system depend on $\mathbf{Q}$, structural geometry and payload. They are described through variation $\mathbf{M}, \mathbf{C}$ and $\mathbf{D}$ matrices because each element of these is a function of them and is given as

$$
\begin{aligned}
& M[i, j]=f\left(\mathbf{Q}, L_{1}, L_{2}, m_{P}\right) ; \\
& D[i, j]=f\left(\mathbf{Q}, L_{1}, L_{2}, m_{P}\right) ; i, j=1, \ldots, n \\
& C[i, j]=f\left(\mathbf{Q}, L_{1}, L_{2}, m_{P}\right) ; \\
& n=4 n_{1}+4 n_{2}+2 .
\end{aligned}
$$

There are many researchers focused on dynamic behaviors under variation of payload [4-6]. However, the effects of structural geometry on dynamic behavior of manipulator have not been clearly considered. In fact, the variation of factorial geometry of links has a huge influence on values elastic displacements which will be considered in the next part.

\section{SIMULATION RESULTS AND DISCUSSION}

The joints of links are constrained to have zero elastic displacements of $q_{1}(t)$ and $q_{2}(t)$ with respect to the fixed axis $O X$. Thus variables $u_{1}, u_{2}, v_{1}$ and $v_{2}$ can be zero. By enforcing these boundary conditions and FEM theory, the generalized coordinate $\mathbf{Q}(t)$ becomes

$$
\mathbf{Q}(t)=\left[\begin{array}{llllllllllll}
q_{1} & u_{3} & u_{4} & \ldots & u_{2 n_{1}+1} & u_{2 n_{1}+2} & q_{2} & v_{3} & v_{4} & \ldots & v_{2 n_{2}+1} & v_{2 n_{2}+2}
\end{array}\right]^{T} .
$$

The respective vector of torque by actuators is given by

$$
\mathbf{F}(t)=\left[\begin{array}{llllllllll}
\tau_{1} & 0 & 0 & \ldots & 0 & \tau_{2} & 0 & 0 & \ldots & 0
\end{array}\right]^{T} .
$$

The dynamic nonlinear equations of two-link flexible manipulator can be derived as followed (21). In this work, the input torque applied signals are $\tau_{1}$ at the hub of link 1 and $\tau_{2}$ at the hub of link 2 and are shown in Fig. 2. The results of equations (21) are output signals which are angular of joints $q_{1}(t), q_{2}(t)$ and elastic displacements at the end of link 1 and link 2. Most of initial values are constant, except that the values of payload and length of links are changed. The initial values of model are given by: $b_{1}=b_{2}=0.02(\mathrm{~m})$; $h_{1}=h_{2}=0.003(\mathrm{~m}) ; A_{1}=b_{1} h_{1}\left(\mathrm{~m}^{2}\right) ; A_{2}=b_{2} h_{2}\left(\mathrm{~m}^{2}\right) ; E_{1}=E_{2}=7,11.10^{10}\left(\mathrm{~kg} / \mathrm{m}^{2}\right) ;$

$\rho_{1}=\rho_{2}=2710\left(\mathrm{~kg} / \mathrm{m}^{3}\right) ; I_{1}=I_{2}=4,5.10^{-11}\left(\mathrm{~m}^{4}\right) ; I_{h 1}=I_{h 2}=5,86.10^{-4}\left(\mathrm{~kg} . \mathrm{m}^{2}\right) ;$ $m_{d c 2}=0,155(\mathrm{~kg}) ; J_{d c 2}=m_{d c 2} L_{1}^{2} / 3\left(\mathrm{~kg} \cdot \mathrm{m}^{2}\right) ; \alpha=0.76 ; \beta=5,6.10^{-5}$;

Case 1: $L_{1}=1(\mathrm{~m}), L_{2}=0.5(\mathrm{~m}), m_{P}=0(\mathrm{~kg}) ; 0.05(\mathrm{~kg}), 0.1(\mathrm{~kg}), 0.15(\mathrm{~kg})$;

Case 2: $L_{1}=L_{2}=1(\mathrm{~m}), m_{P}=0(\mathrm{~kg}) ; 0.05(\mathrm{~kg}) ; 0.01(\mathrm{~kg}) ; 0.15(\mathrm{~kg}) ;$

Case 3: $L_{1}=0.5(\mathrm{~m}), L_{2}=1(\mathrm{~m}), m_{P}=0(\mathrm{~kg}) ; 0.05(\mathrm{~kg}) ; 0.1(\mathrm{~kg}) ; 0.15(\mathrm{~kg})$. 

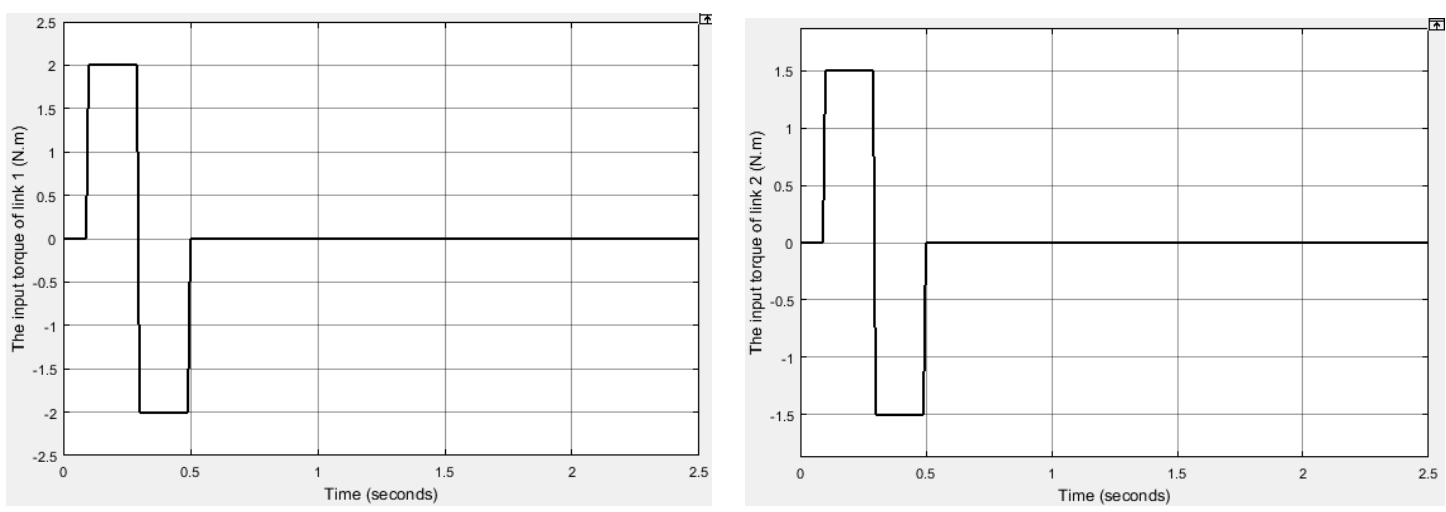

Fig. 2. The torques input of link 1 and link 2

To determine elastic displacements under variation of payload and length of links on a two flexible links manipulator, a computer program was written within MATLABSIMULINK environment which is shown in Fig. 3.

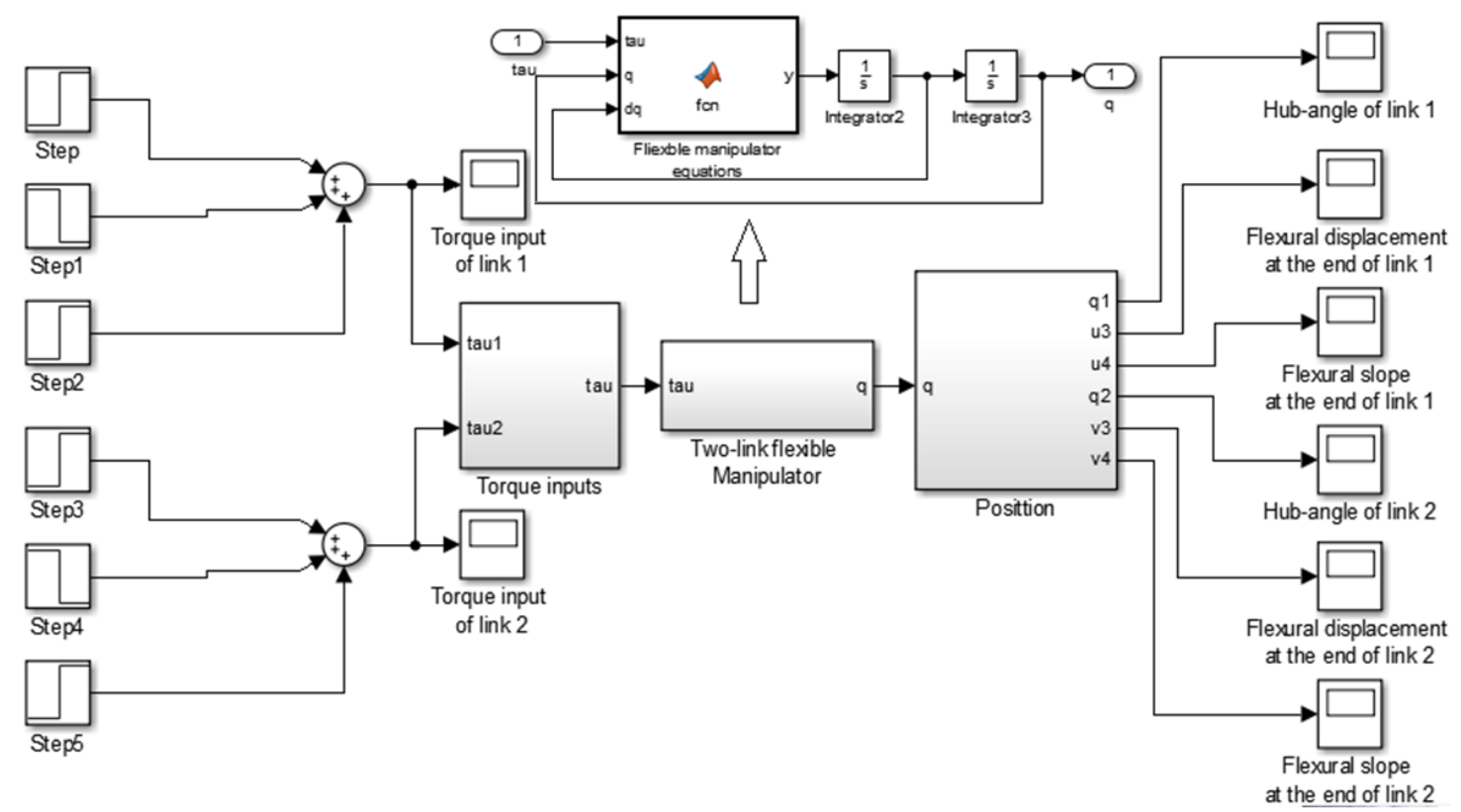

Fig. 3. System block diagram in SIMULINK

Time domain results of the effects of varied payload for the case 1 are shown in Fig. 4.The length of link 1 is longer than the length of link $2\left(L_{1}=1 ; L_{2}=0.5(\mathrm{~m})\right)$. The lengths ratio is $2: 1$. The maximum value of flexural displacement is $0.082(\mathrm{~m})$ and slope displacement is $0.25(\mathrm{rad})$, respectively, $m_{P}=0(\mathrm{~g})$, respectively. They are decreasing when the payload increases. In case 2, the lengths of link 1 and link 2 are equal. The values of flexural and slope displacement are shown in Fig. 5. The maximum flexural value is $0.5(\mathrm{~m})$ and slope value is 0.8 (rad). Elastic displacements are decreased with 

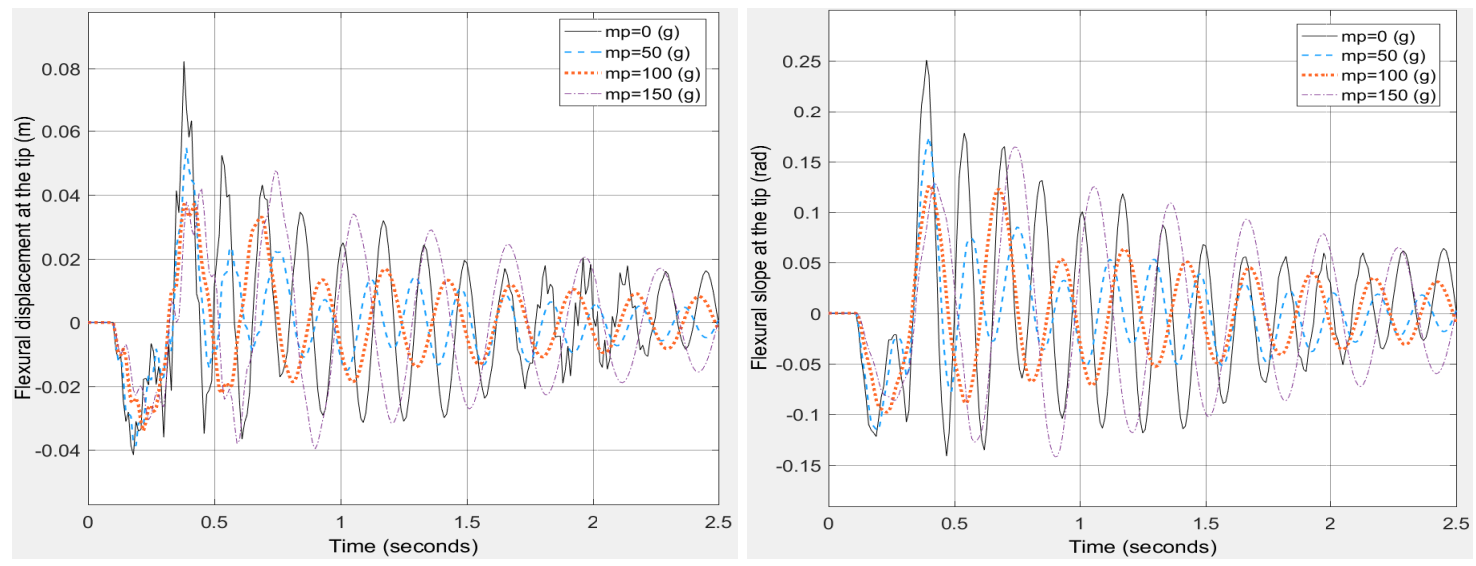

Fig. 4. The flexural and slope displacement at the end-effector in Case $1\left(L_{1}=1 ; L_{2}=0.5(\mathrm{~m})\right)$

increasing payloads. They tended to increase than case 1 while the ratio between length of link 1 and link 2 is decreased. Finally, Fig. 6 shows the values of elastic displacements at the end point of link 2 in case $3\left(L_{1}=0.5(\mathrm{~m}) ; L_{2}=1(\mathrm{~m})\right)$. The ratio $\left(L_{1}: L_{2}\right)$ is $(1: 2)$. The values of elastic displacements are the biggest in all of three cases. But they are still decreased with increasing payloads. The results can be summarized in Tab. 1.
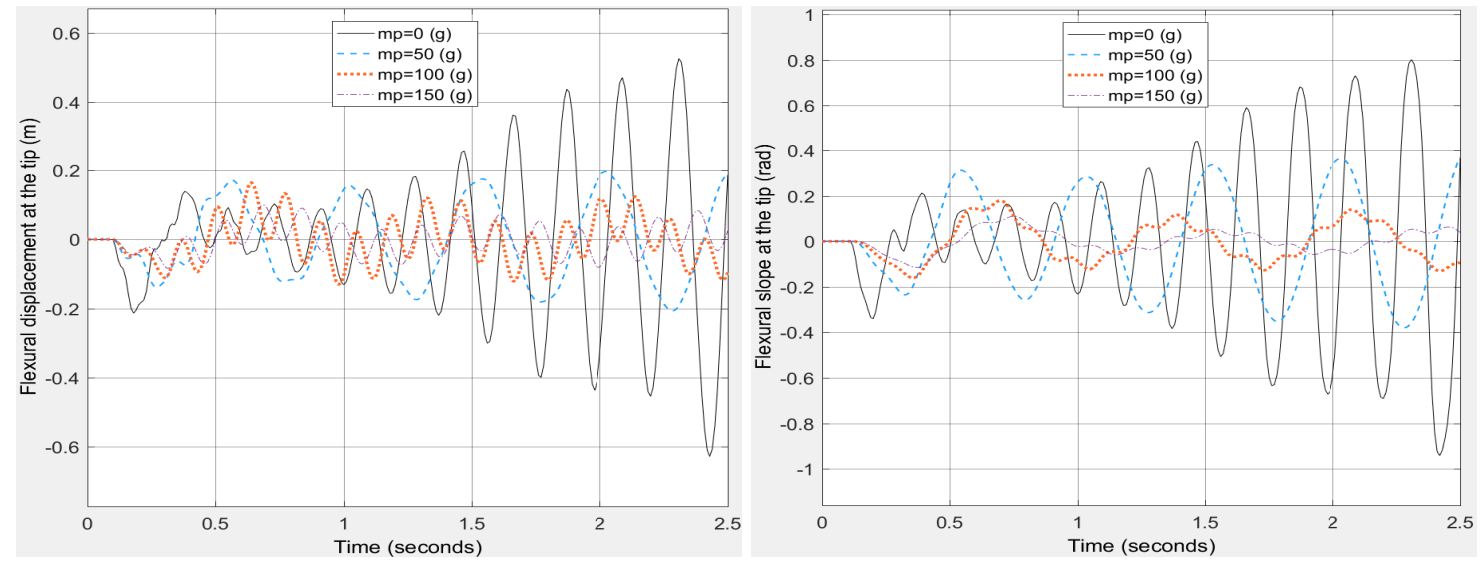

Fig. 5. The elastic displacements at the end-effector in Case $2\left(L_{1}=1 ; L_{2}=1(\mathrm{~m})\right)$

By comparing the results presented in Tab. 1, it is noted that when the length ratio between link 1 and link 2 is decreasing the elastic displacement values increase while the payload is constant. Furthermore, these values are not much different. When the length ratio is constant the elastic displacements decrease and the system vibrates in long time with increasing payloads in all of three cases. Besides, the values of elastic displacements at the end point of link 2 are smallest while the length of link 1 is longer than length of link 2. 

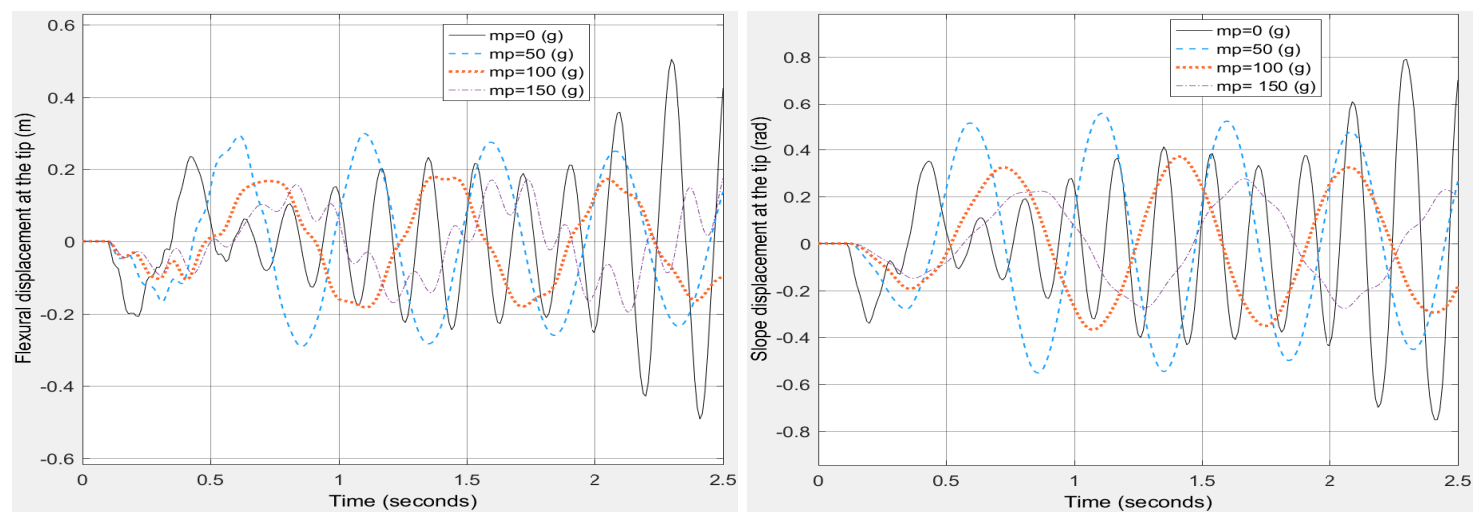

Fig. 6. The elastic displacements at the end-effector in Case $3\left(L_{1}=0.5 ; L_{2}=1(\mathrm{~m})\right)$

Table 1. Effects of variation of payloads and length of links for elastic displacements values at the end point of link 2

\begin{tabular}{|c|c|c|c|c|c|c|}
\hline \multirow{2}{*}{$\begin{array}{c}\text { Payloads } \\
(\mathrm{g})\end{array}$} & \multicolumn{4}{|c|}{ Maximum flexural displacement $(\mathrm{m})$} & \multicolumn{3}{|c|}{ Maximum slope displacement (rad) } \\
\cline { 2 - 7 } & $\begin{array}{c}\text { Case 1 } \\
\left(L_{1}<L_{2}\right)\end{array}$ & $\begin{array}{c}\text { Case 2 } \\
\left(L_{1}=L_{2}\right)\end{array}$ & $\begin{array}{c}\text { Case 3 } \\
\left(L_{1}>L_{2}\right)\end{array}$ & $\begin{array}{c}\text { Case 1 } \\
\left(L_{1}<L_{2}\right)\end{array}$ & $\begin{array}{c}\text { Case 2 } \\
\left(L_{1}=L_{2}\right)\end{array}$ & $\begin{array}{c}\text { Case 3 } \\
\left(L_{1}>L_{2}\right)\end{array}$ \\
\hline 0 & 0.082 & 0.5 & 0.6 & 0.25 & 0.8 & 0.8 \\
\hline 50 & 0.055 & 0.21 & 0.3 & 0.17 & 0.35 & 0.55 \\
\hline 100 & 0.04 & 0.16 & 0.18 & 0.125 & 0.18 & 0.38 \\
\hline 150 & 0.05 & 0.1 & 0.2 & 0.16 & 0.12 & 0.28 \\
\hline
\end{tabular}

\section{CONCLUSION}

A nonlinear dynamic model of a two-link flexible manipulator incorporating structural damping, hub inertia, Coriolis and centrifugal forces and payload has been presented. The dynamic equations are built based on finite element method in LagrangeEuler approach. The determination of the elastic displacement plays an essential role in the consideration of the system dynamic behavior under the variation of the payload and the link length ratio. These results are very helpful and important in the mechanical design of the links and in the development of effective control algorithms for the flexible manipulators with varying payload.

\section{REFERENCES}

[1] L. T. Wang and B. Ravani. Dynamic load carrying capacity of mechanical manipulators - part I: Problem formulation. Journal of Dynamic Systems, Measurement, and Control, 110, (1), (1988), pp. 46-52. doi:10.1115/1.3152647.

[2] L. T. Wang and B. Ravani. Dynamic load carrying capacity of mechanical manipulators - part II: Computational procedure and applications. Journal of Dynamic Systems, Measurement, and Control, 110, (1), (1988), pp. 53-61. doi:10.1115/1.3152648. 
[3] M. H. Korayem, M. Nazemizadeh, and H. R. Nahooji. Dynamic load carrying capacity of flexible manipulators using finite element method and pontryagins minimum principle. Journal of Optimization in Industrial Engineering, 6, (12), (2013), pp. 17-24.

[4] F. Y. Wang and J. L. Russell. Optimum shape construction of flexible manipulators with tip loads. In Proceedings of the 31st Conference on Decision and Control, Tucson, Arizona, (1992). IEEE, pp. 311-316.

[5] S. S. Ge, T. H. Lee, and G. Zhu. A nonlinear feedback controller for a single-link flexible manipulator based on a finite element model. Journal of Robotic Systems, 14, (3), (1997), pp. 165178. doi:10.1002/(sici)1097-4563(199703)14:3<165::aid-rob2>3.0.co;2-p.

[6] M. A. Ahmad, Z. Mohamed, and N. Hambali. Dynamic modelling of a two-link flexible manipulator system incorporating payload. In Proceedings of the 3rd IEEE Conference on Industrial Electronics and Applications. IEEE, (2008), pp. 96-101. doi:10.1109/iciea.2008.4582487.

[7] M. P. P. Reddy and J. Jacob. Accurate modeling and nonlinear finite element analysis of a flexible-link manipulator. International Journal of Mechanical, Aerospace, Industrial and Mechatronics Engineering, 8, (1), (2014), pp. 165-170.

[8] M. S. Alam. Dynamic modelling of flexible manipulator system using genetic algorithm. Dhaka University Journal of Science, 60, (2), (2012), pp. 239-245. doi:10.3329/dujs.v60i2.11526.

[9] J. Jafari, M. Mirzaie, and M. Zandbaf. Mathematical modeling and nonlinear dynamic analysis of flexible manipulators using finite element method. Universal Journal of Non-linear Mechanics, 1, (2013), pp. 56-61.

[10] K. Ibrahim, A. Aly, and A. A. Ismail. Mode shape analysis of a flexible robot arm. International Journal of Control, Automation and Systems, 1, (2), (2013), pp. 8-15.

[11] G. V. Narayana and R. P. Modeling and control flexible link manipulator using linearization techniques. International Journal of Current Engineering and Technology, 3, (2), (2013), pp. 611616.

[12] J. F. Peza-Solís, G. Silva-Navarro, and N. R. Castro-Linares. Trajectory tracking control in a single flexible-link robot using finite differences and sliding modes. Journal of Applied Research and Technology, 13, (1), (2015), pp. 70-78. doi:10.1016/s1665-6423(15)30006-7.

[13] M. H. Korayem, M. Haghpanahi, and H. R. Heidari. Maximum allowable dynamic load of flexible manipulators undergoing large deformation. Mechanical Engineering, 17, (1), (2010), pp. 61-74.

[14] A. A. Ata, W. F. Fares, and M. Y. Saadeh. Dynamic analysis of a two-link flexible manipulator subject to different sets of conditions. Procedia Engineering, 41, (2012), pp. 1253-1260. doi:10.1016/j.proeng.2012.07.308.

[15] M. O. Tokhi, Z. Mohamed, and M. H. Shaheed. Dynamic characterisation of a flexible manipulator system. Robotica, 19, (5), (2001), pp. 571-580. doi:10.1017/s0263574700003209.

[16] T. I. Thinh and N. N. Khoa. Finite element method. Hanoi University of Science and Technology, (2007). (in Vietnamese). 